\title{
Synthesis of a Novel Benzoxazine Monomer-Intercalated Montmorillonite and the Curing Kinetics of Polybenzoxazine/Clay Hybrid Nanocomposites
}

\author{
DING-RU YEI, HUEI-KUAN FU, WEN-YI CHEN, FENG-CHIH CHANG \\ Institute of Applied Chemistry, National Chiao-Tung University, Hsin-Chu, Taiwan, Republic of China
}

Received 1 August 2005; revised 12 October 2005; accepted 16 October 2005

DOI: 10.1002 / polb.20693

Published online in Wiley InterScience (www.interscience.wiley.com).

\begin{abstract}
Polybenzoxazine (PBZ), which has a structure similar to that of phenolic resin, is formed through the thermal self-curing of benzoxazine, that is through a heterocyclic ring opening reaction that requires no catalyst and releases no condensation byproducts. We have used the solvent blending method to prepare PBZ/clay nanocomposites possessing various clay contents. We synthesized a monofunctional benzoxazine monomer (MBM) and then treated the clay with this intercalation agent. The results of X-ray diffraction (XRD) analysis indicated that MBM intercalated into the galleries of the clay; the nanocomposite possessed an exfoliated structure at $3 \%$ clay content. To better understand the curing kinetics of the PBZ/clay nanocomposites, we performed dynamic and isothermal differential scanning calorimetry (DSC) measurements. We describe the thermodynamics of the curing process, using all three of the Kissinger, Ozawa, and Kamal models. The Kissinger and Ozawa methods gave fairly close results for the calculated activation energies, which decreased upon increasing the clay content. The Kamal method, based on an autocatalytic model, suggested a total reaction order of between 2.4 and 2.8. The glass transition temperature $\left(T_{\mathrm{g}}\right)$ decreased upon increasing the clay content. Thermogravimetric analysis (TGA) indicated that the nanocomposites have higher decomposition temperatures than does the pristine PBZ; this finding suggests an enhancement in their thermal stability. ( 2005 Wiley Periodicals, Inc. J Polym Sci Part B: Polym Phys 44: 347-358, 2006
\end{abstract}

Keywords: montmorillonite; nanocomposites; polybenzoxazine

\section{INTRODUCTION}

Polybenzoxazine (PBZ) is a newly developed thermosetting resin that has a number of interesting properties. It is a novel type of phenolic resin that was developed to overcome the shortcomings of the traditional novolac and resole polymers. Although PBZ possesses the excellent properties commonly associated with the tradi-

Correspondence to: F. C. Chang (E-mail: changfc@mail.nctu. edu.tw)

Journal of Polymer Science: Part B: Polymer Physics, Vol. 44, 347-358 (2006) @ 2005 Wiley Periodicals, Inc. tional phenolic resins—-such as heat, electrical, and flame resistance-it also displays a number of unique characteristics, including excellent dimensional stability (resulting from the ringopening polymerization mode), improved toughness, a stable dielectric constant, and low moisture absorption. In addition, PBZ derivatives can be synthesized from inexpensive raw materials, can be cured without requiring strong acid or base catalysis, and do not release any by-products during polymerization; thus, they are considered to be attractive candidates for many applications. ${ }^{1-7}$

Polymer/clay nanocomposites, in which the layered silicates of the clay become dispersed in 
the polymeric matrix, are a new class of composite materials. As a result of the dispersion of the clay on the nanoscale, such nanocomposites often exhibit outstanding improvements in their properties-including increased modulus, strength, thermal stability, and solvent resistance and decreased gas permeability and flammability ${ }^{8-25}$ relative to those of conventional fiber or fillerfilled composites. The layered silicate that is most widely used for the preparation of polymer/clay nanocomposites is montmorillonite (MMT).

MMT is a multilayer silicate mineral that naturally possesses inorganic cations within its galleries to balance the charge of the oxide layers in a hydrophilic environment. The ion exchange of these cations with organic ammonium ions affords hydrophobic environment within the galleries of the organically modified MMT (OMMT). ${ }^{15}$ The organophilic galleries of OMMT enhance the compatibility of the clay with polymers, ${ }^{15}$ improve the dispersion of the silicate layers into the matrix, ${ }^{12}$ and assist the penetration of monomers or polymers into the galleries. ${ }^{22}$ In addition, the organic ammonium cations can provide functional groups that react or interact with the monomer or polymer units to improve the interfacial strength between the reinforcement and the polymer matrix. ${ }^{18}$ The degree of dispersion of clay nanolayers and the resulting morphology of the nanocomposite depend on a number of factors, including the mixing method (melt or solvent), temperature, time, the choice of solvent, and its concentration, the steric size of the monomer or polymer, the choice of intercalation agent, and the yield of the ion exchange process.

In this study, we synthesized a monofunctional benzoxazine monomer (MBM) that behaves as an intercalation agent and used it subsequently to prepare $\mathrm{PBZ} /$ clay hybrid nanocomposites. We expected that this intercalation agent would undergo ring opening polymerization with benzoxazine matrix to promote the formation of an exfoliated nanocomposite structure. Scheme 1 displays the structures of the benzoxazine monomer and PBZ. To study the kinetics of the development process, we performed isothermal and dynamic differential scanning calorimetry (DSC) experiments. We have used two dynamic kinetic models and one isothermal model to study the kinetics of the curing of the PBZ/clay nanocomposites. We used both X-ray diffraction (XRD) and transmission electron microscopy (TEM) to characterize the structure of the clay, and characterized the thermal properties of the PBZ/clay nano-

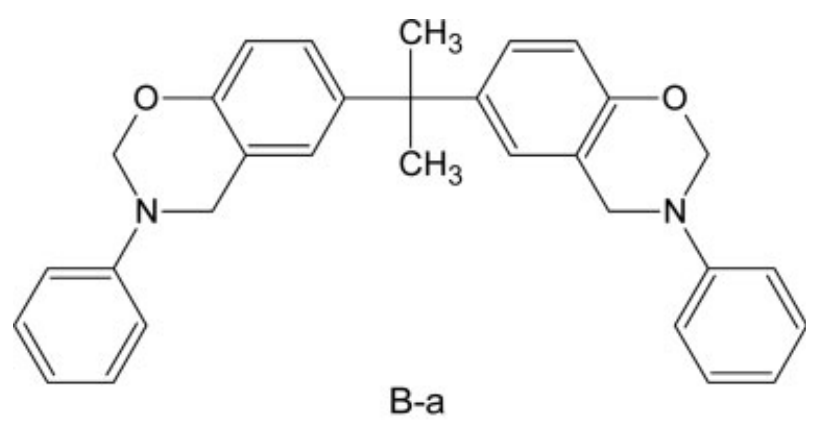

Thermal treatment

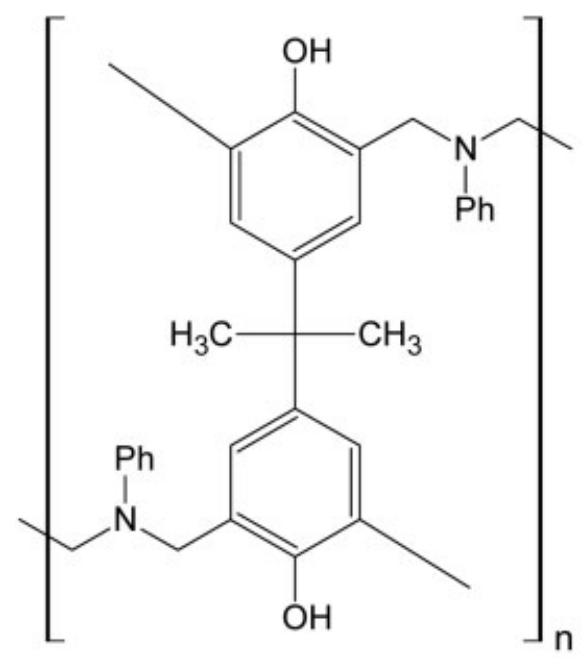

\section{Polybenzoxazine (PBZ)}

Scheme 1. Preparation of PBZ.

composites through thermogravimetric analysis (TGA).

\section{EXPERIMENTAL}

\section{Materials}

Bis(3-phenyl-3,4-dihydro-2H-1,3-benzoxazinyl) isopropane (B-a), a PBZ precursor, was supplied by Shikoku Chemicals. Phenol was purchased from SHOWA Chemical, Japan. Formalin and $\mathrm{N}, \mathrm{N}$-dimethyl-1,3-propanediamine were obtained from Acros Organics, USA. Tetrahydrofuran and dioxane were purchased from Aldrich Chemical, 


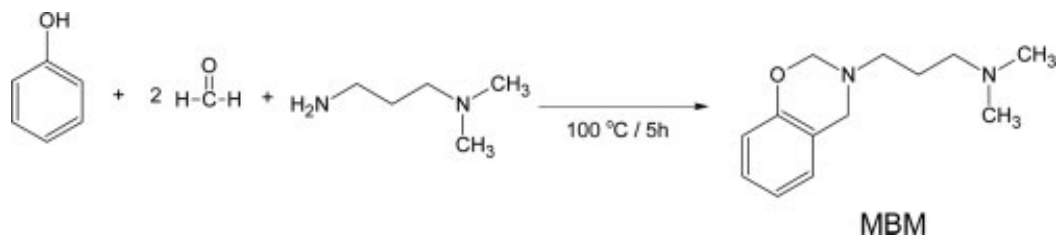

Scheme 2. Preparation of the MBM.

USA. Pristine Na-MMT was provided by Telekal, Taiwan.

\section{Synthesis of MBM}

MBM was synthesized according to the procedure presented in Scheme 2. Formalin (37\%, $16.23 \mathrm{~g}$, $200 \mathrm{mmol})$ and dioxane $(100 \mathrm{~mL})$ were mixed in a 500 -mL flask that was cooled in an ice bath. $N, N$ Dimethyl-1,3-propanediamine (10.2 g, $100 \mathrm{mmol}$ ) in dioxane $(100 \mathrm{~mL})$ was added portionwise to this solution. The solution was then stirred for 30 min while maintaining the temperature below $5{ }^{\circ} \mathrm{C}$. Thereafter, a solution of phenol $(9.41 \mathrm{~g}$, $100 \mathrm{mmol})$ in dioxane $(100 \mathrm{~mL})$ was added. The solution was heated under reflux at $110{ }^{\circ} \mathrm{C}$ for $4 \mathrm{~h}$. Removal of the solvent in a rotary evaporator at $50{ }^{\circ} \mathrm{C}$ gave a viscous residue that was dissolved in ether $(200 \mathrm{~mL})$ and washed several times with $3 \mathrm{~N}$ sodium hydroxide solution and then with distilled water. The ether solution was dried (anhydrous sodium sulfate); evaporation of the solvent under vacuum afforded a pale-yellow viscous fluid.

\section{Preparation of MBM-Modified Clays}

Prewashed clay $(1 \mathrm{~g})$ and water $(50 \mathrm{~mL})$ were placed into a 100-mL, two-neck, round-bottom flask and stirred continuously for $4 \mathrm{~h}$. In a separate flask, $10 \%$ hydrochloric acid $(5 \mathrm{~mL})$ was added to a solution of MBM $(0.4 \mathrm{~g})$ in THF $(5 \mathrm{~mL})$; this mixture was stirred for $1 \mathrm{~h}$ before being added to the suspended clay and the then stirred overnight. The mixture was filtered, washed several times with deionized water, and then dried overnight in a vacuum oven at room temperature.

\section{Preparation of PBZ/Clay Nanocomposites}

Benzoxazine (B-a) and the MBM-modified clay were blended together at various weight ratios. An example of the solvent method is presented here for the preparation of a sample possessing a $3 \%$ inclusion of MBM-modified clay: MBM-modi- fied clay $(0.3 \mathrm{~g})$ was dispersed in THF $(20 \mathrm{~mL})$ at room temperature for $2 \mathrm{~h}$. B-a (9.7 g) was added to the MBM-modified clay suspension and then the sample was stirred at room temperature overnight to form a well-dispersed mixture. The THF was evaporated under vacuum. The prepared samples were used for isothermal and dynamic curing experiments employing DSC. Subsequently, the samples were cured at 130, 160, 180, and $200{ }^{\circ} \mathrm{C}$ for $2 \mathrm{~h}$ each in an oven under an atmosphere of air to obtain the PBZ/clay nanocomposites.

\section{Isothermal Curing}

The samples were placed in the $\mathrm{Al}$ cell at room temperature and then heated quickly to a preset temperature for each isothermal experiment. The instrument achieved a stable state about $60 \mathrm{~s}$ after reaching the setting temperature; the data were recorded immediately. When the DSC exothermic peak reached the baseline level, the isothermal curing process was complete and the heat of reaction was measured by integrating the area of the exothermic peak. These isothermal curing curves were used to evaluate the kinetic parameters of the curing reaction.

\section{Dynamic Curing}

The nonisothermal experiments were performed at heating rates of $2.5,5,10$, and $20^{\circ} \mathrm{C} / \mathrm{min}$ over the temperature range from 30 to $320{ }^{\circ} \mathrm{C}$; the area of exothermic peak was integrated. We assumed that the total recorded heat of the reaction represented the total heat evolved for the complete curing process at the different curing rates.

\section{Instrumentation}

XRD spectra were collected on an M18XHF-SPA XRD instrument (MacScience, Japan), using Co $\mathrm{K} \alpha$ radiation; Bragg's law $(\lambda=2 d \sin \theta)$ was used to compute the spacing. Transmission electron 


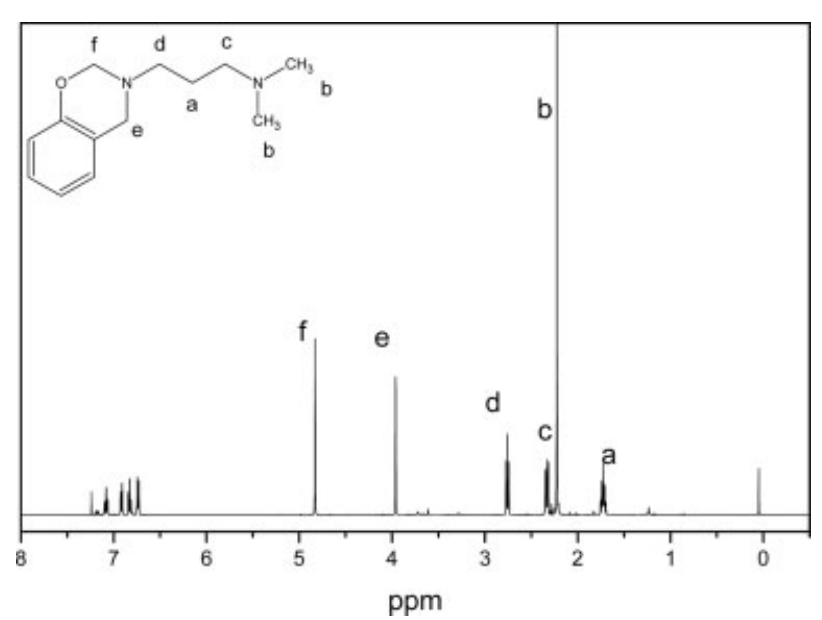

Figure 1. ${ }^{1} \mathrm{H}$ NMR spectrum of MBM.

microscopy (TEM) images of the composites were obtained at $100 \mathrm{kV}$ using a Hitachi H-7500 electron microscope. The sample was ultramicrotomed at room temperature using a diamond knife on a Leica Ultracut UCT microtome to provide $80-\mathrm{nm}$ thick sections. The contrast between the layered silicates and the polymer phase was sufficient for imaging, and therefore, no heavy metal staining was required prior to imaging. Thermogravimetric analyses (TGA) were performed using a TA Instruments thermal analyzer under a $40 \mathrm{~mL} / \mathrm{min}$ flow of nitrogen gas at a scan rate of $20{ }^{\circ} \mathrm{C} / \mathrm{min}$ over the temperature range from 30 to $800{ }^{\circ} \mathrm{C}$. A Du Pont (DSC-9000) differential scanning calorimeter (DSC) was used to measure both the glass transition temperature $\left(T_{\mathrm{g}}\right)$ of the $\mathrm{PBZ} /$ clay nanocomposite and the kinetic parameters of the curing reaction.

\section{RESULTS AND DISCUSSION}

\section{Preparation of MBM}

MBM was prepared according to the procedure presented in Scheme 2. Initially, dimethyl-1,3propanediamine was reacted with formalin and phenol in dioxane. The crude product was dissolved in ether and washed with aqueous $\mathrm{NaOH}$ to remove any phenolic oligomers; evaporation of the solvent afforded MBM as a viscous yellow product. We used ${ }^{1} \mathrm{H}$ NMR spectroscopy to confirm the chemical structure of MBM (Fig. 1). The three multiplets at $1.75,2.33$, and $2.75 \mathrm{ppm}$ are typical for the $\mathrm{CH}_{2}$ protons of the allyl group. We assign the peak at $2.23 \mathrm{ppm}$ for the $\mathrm{N}\left(\mathrm{CH}_{3}\right)_{2}$ protons. The characteristic protons of oxazine ring, at 3.95 and $4.83 \mathrm{ppm}$, are assigned to $\mathrm{ArCH}_{2} \mathrm{~N}$ and $\mathrm{OCH}_{2} \mathrm{~N}$ units, respectively. The aromatic protons appear as a multiplet at $6.73-7.23 \mathrm{ppm}$.

\section{X-Ray Diffractions}

We employed XRD to characterize the layered structures of the modified clays and PBZ/clay nanocomposites; changes in the value of $2 \theta$ reflect changes in the gallery distance of the clay. Figure 2 displays the XRD results of the MBM-intercalated clay. The pristine clay sample exhibits a peak at $6.14^{\circ}$, which corresponds to a basal space of 1.43 $\mathrm{nm}$. The insertion of the MBM between the galleries of the clay increased the $d$ spacing from 1.43 to $1.84 \mathrm{~nm}$. This result indicates that the MBM was intercalated successfully into the galleries of the clay nanoparticles. Scheme 3 displays the clay intercalated by the MBM. We calculated the interlamellar distance, which is indicated in this Scheme, by using the expression: $\Delta d=d$ spacing - thickness of one platelet ( $\sim 9 \AA)$. For the clay intercalated with the MBM, the distance between two adjacent clay plates was $9.4 \AA$; this value is close to the size of one MBM molecule.

Figure 3 presents the XRD patterns for the nanocomposites formed when varying the clay contents. We detected no peak for nanocomposite containing 3\% clay content; this observation implies that this sample possesses an exfoliated structure. We did observe, however, a peak at about $2 \theta=3.5^{\circ}$ for each of the samples containing 6,10 , and $15 \%$ clay content. These data suggest the presence of the intercalated structure, but these measurements alone were not conclusive

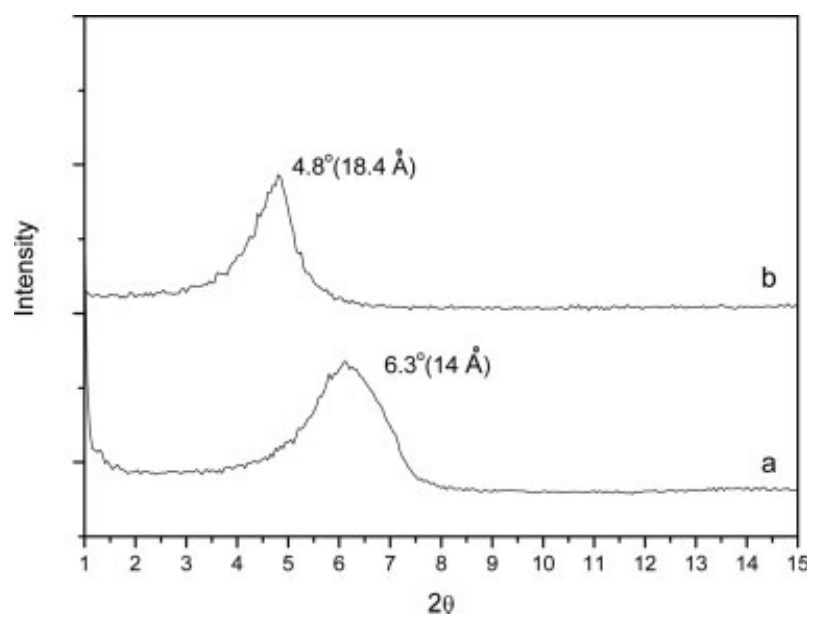

Figure 2. XRD patterns of (a) pure clay and (b) the clay intercalated by MBM. 


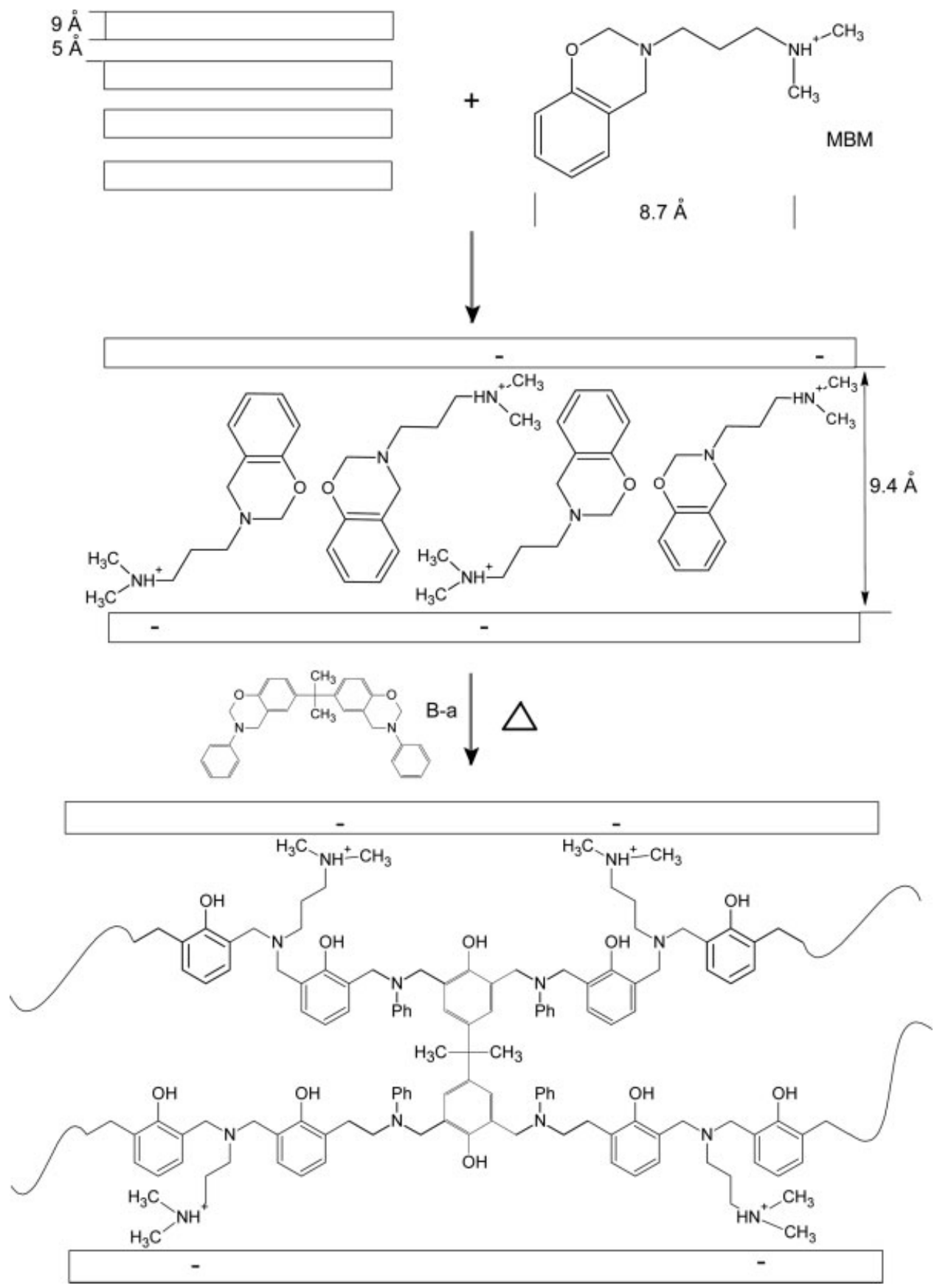

Scheme 3. Schematic illustration of clay intercalated by the MBM and polybenzoxaine.

for determining the true structures and distributions of the silica platelets; thus, we turned our attention to TEM measurements.

\section{TEM Measurements of the Nanocomposites}

Figure 4 displays TEM images of the 3 and $6 \%$ clay nanocomposites. These images indicate the dispersion of the clay layers within the polymer. The dark lines represent the clay layers that were positioned perpendicular to the sample's surface. For the 3\% clay nanocomposite [Fig. 4(a)], each clay layer was isolated and distributed evenly within the PBZ matrix; average distance from a particle to a particle seems to exceed $100 \mathrm{~nm}$. This situation implies that a mixture of interca- 


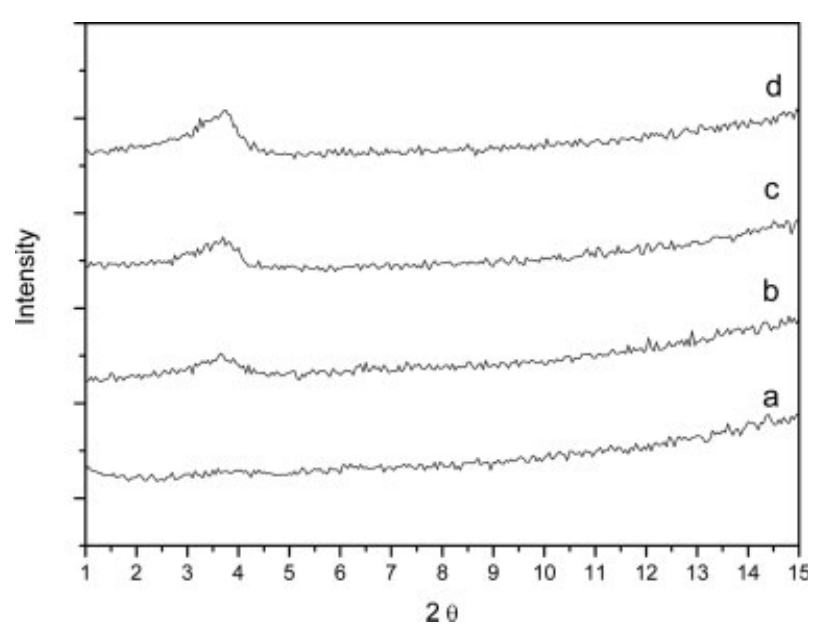

Figure 3. XRD patterns of the (a) 3\%, (b) 6\%, (c) $10 \%$, and (d) $15 \%$ clay nanocomposites.

lated and exfoliated structures had occurred. The platelet layers we observe for the $6 \%$ clay nanocomposite are a mixture of fully exfoliated and intercalated structures.

\section{Investigating the Curing Behavior of PBZ/Clay Nanocomposites Using DSC}

DSC is an attractive technique because it can provide detailed information regarding the cure mechanism and the preferred temperature during the formation of three-dimensional networks in materials. In addition, the apparatus is simple to use, data are obtained quickly, and only small quantities of samples are required. DSC is employed widely to elucidate the key parameters of the curing process, such as the extent and rate of chemical conversion and the glass transition temperature, from both isothermal and dynamic DSC experiments.

Figure 5 presents the dynamic exothermal curves, obtained at a heating rate $20{ }^{\circ} \mathrm{C} / \mathrm{min}$, of the samples possessing the various clay contents. We observed a single exothermic peak for each curing system, but the sharp exothermic peak for the pure PBZ system occurred at a relatively higher temperature. For the PBZ/clay systems, the exothermic peak was smooth and broad, and it shifted to a lower temperature upon increasing the clay content. These results suggest that the curing reaction in the pure PBZ system was delayed to a higher temperature.

Figure 6 presents the DSC exothermic curves of pure PBZ recorded at heating rates $2.5,5,10$, and $20{ }^{\circ} \mathrm{C} / \mathrm{min}$. We observe that the curing temperature (i.e., the peak exotherm temperature, $T_{\mathrm{p}}$ ) increased upon increasing the heating rate. We investigated these exotherms using the dynamic kinetic method to obtain the activation energy $\left(E_{\mathrm{a}}\right)$ and other related parameters. Figure 7 displays the reaction rates of pure PBZ as a function of the curing time for different curing temperatures. We cured all of the samples without adding any catalyst and observed only a single exothermic peak in each DSC curve. In other words, this heterocyclic ring opening curing reaction involves a single exothermic chemical process. As expected, we found that a higher curing temperature increased the curing rate and decreased the curing time.

Figure 8 provides a plot of the conversion $(\alpha)$ as a function of the isothermal curing time for the samples containing various clay contents. When we increased the clay content from 0 to $15 \%$, the
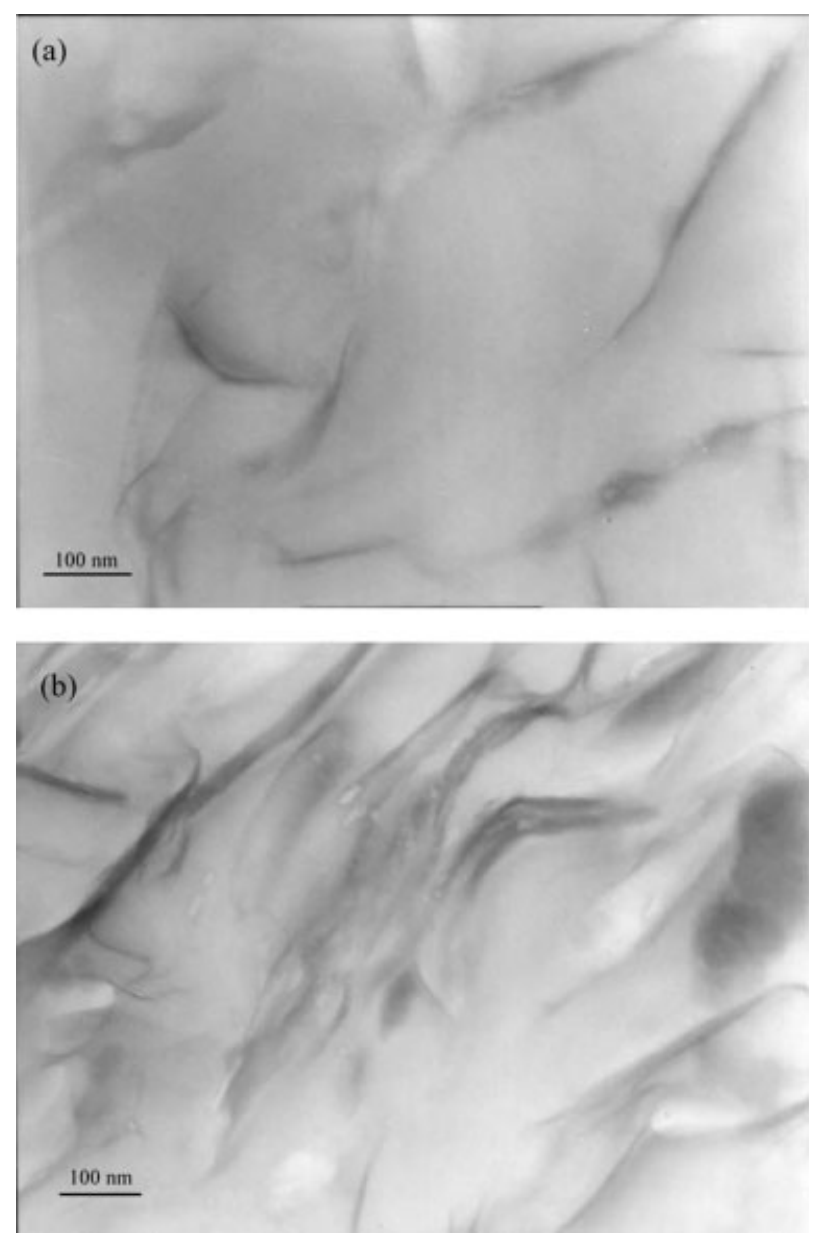

Figure 4. TEM micrographs of the (a) $3 \%$ and (b) $6 \%$ clay nanocomposites. 


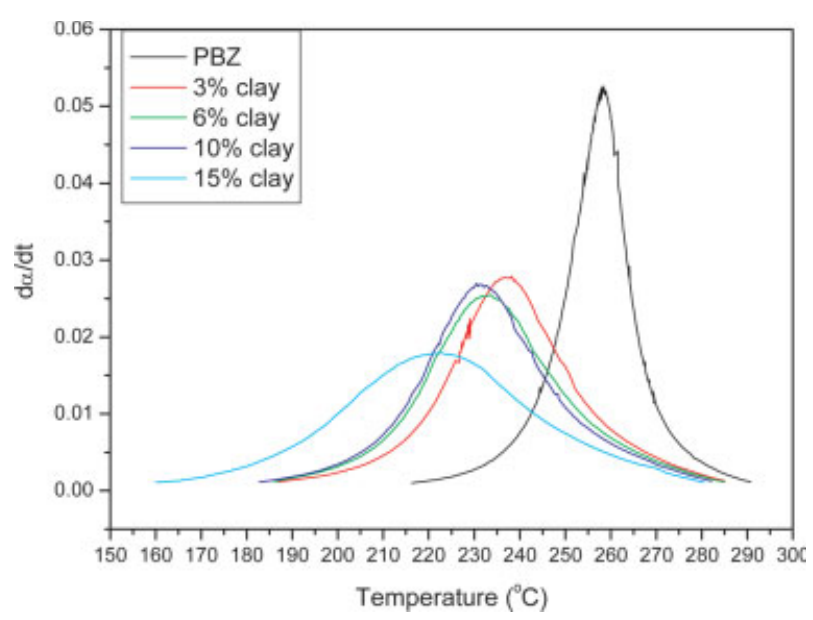

Figure 5. Dynamic exothermal curves of the PBZ/ clay nanocomposites recorded at a heating rate of $20{ }^{\circ} \mathrm{C} / \mathrm{min}$.

conversion decreased from 0.67 to 0.43 . In other words, higher clay content tends to retard the ring opening polymerization of benzoxazine as a result of the clay providing a steric barrier. At initial stage, the clay can catalyze the ring opening polymerization of the benzoxazine, but the clay also creates a steric barrier at final stage of reaction. Figure 9 displays plots of the reaction rate as a function of the conversion at various curing temperatures. The maximum reaction rates occurred at conversions between $17 \%\left(180{ }^{\circ} \mathrm{C}\right)$ and $23 \%\left(220^{\circ} \mathrm{C}\right)$. These conversions are fairly close to those found typically for autocatalyzed reactions: the maximum reaction rate is usually obtained between 20 and $40 \%$ conversion. ${ }^{26-28}$

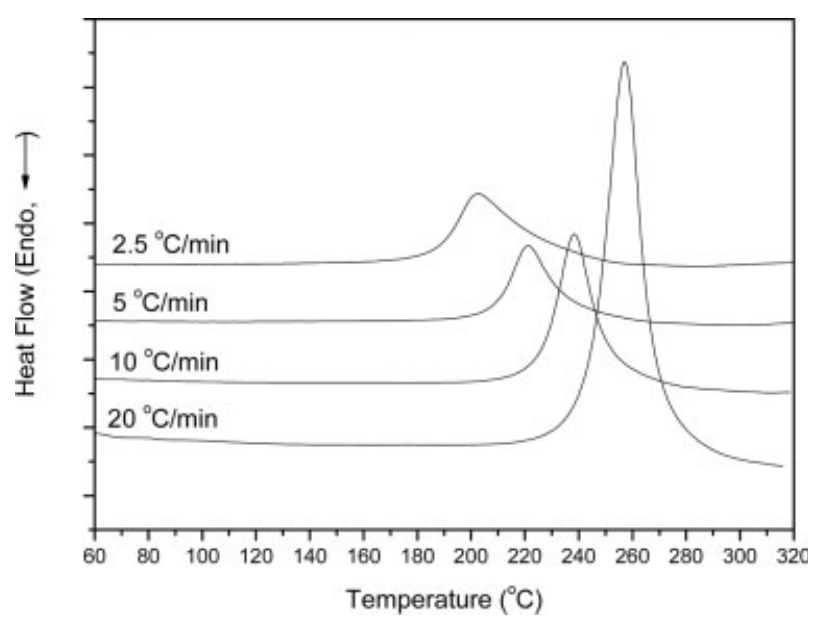

Figure 6. Dynamic DSC exothermic curves of pure $\mathrm{PBZ}$ recorded at different scan rates.

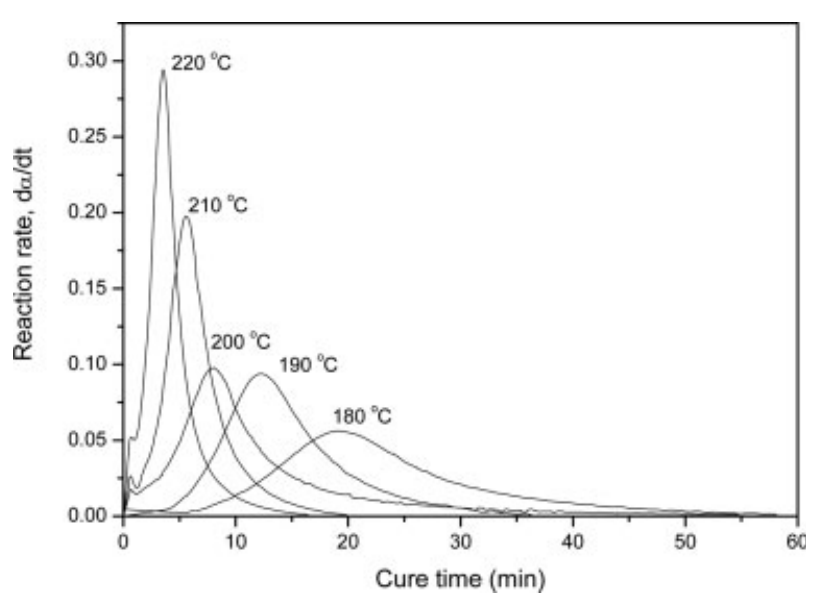

Figure 7. Plots of reaction rate versus curing time for pure PBZ at different curing temperatures.

\section{Kinetic Analysis}

Kinetic analyses are performed most commonly ${ }^{29}$ using three kinetic models: the Kissinger ${ }^{30}$ and Osawa $^{31}$ methods are suitable for dynamic kinetic analyses, while the Kamal ${ }^{32}$ method is suitable for isothermal kinetic analyses (autocatalytic model).

The extent of reaction $(\alpha)$ is proportional to the heat generated during the reaction. The reaction rate is a function of conversion, which can be expressed by the general law provided in eq 1 :

$$
\frac{\mathrm{d} \alpha}{\mathrm{d} t}=k(T) f(\alpha)
$$

where $t$ is the time, $k(\mathrm{~T})$ is the rate constant, and $f(\alpha)$ is a function of the dependence of conversion.

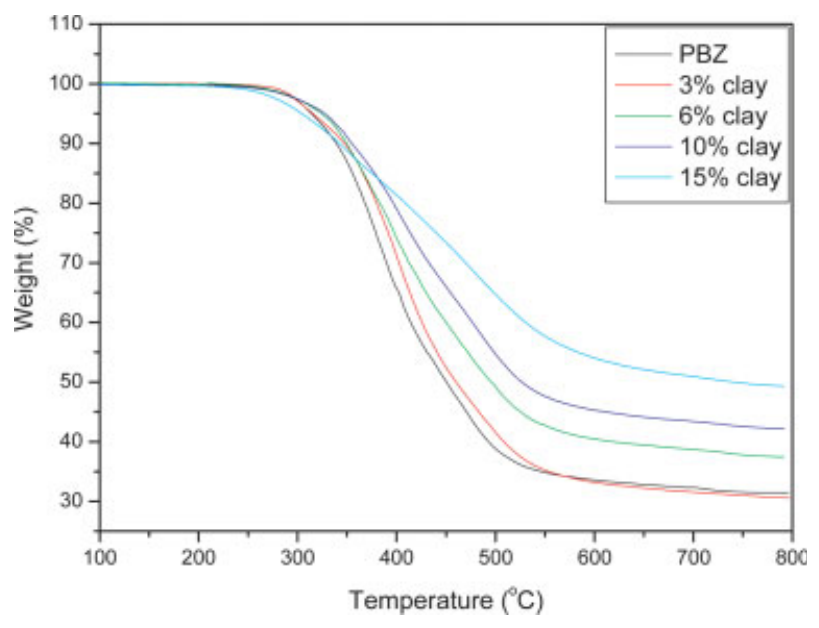

Figure 12. TGA curves of the PBZ/clay nanocomposites recorded under a nitrogen atmosphere. 


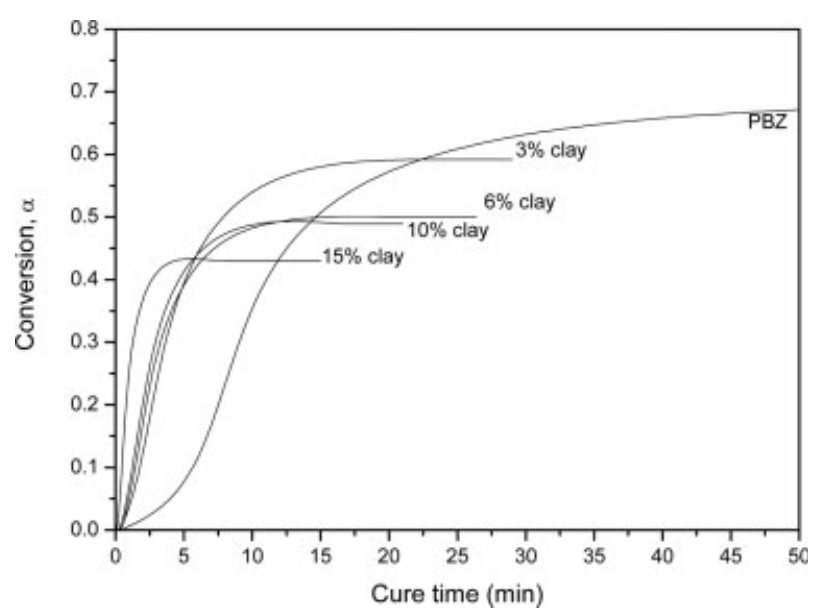

Figure 8. Plots of conversion as a function of cure time for the PBZ/clay nanocomposites cured at isothermal curing temperature of $200{ }^{\circ} \mathrm{C}$.

By integrating the eq 1 , a new equation can be obtained:

$$
g(\alpha)=\int_{0}^{\alpha} \frac{\mathrm{d} \alpha}{f(\alpha)}=k(T) t
$$

where $g(\alpha)$ is the integrated form of the conversion-dependent function.

\section{Dynamic Kinetic Method}

The nonisothermal process can be modeled using both the single and multiple heating rate methods. The multiple heating rate method was proposed by Kissinger and Ozawa. We used these two methods in this study-rather than the other nonisothermal methods-because they do not

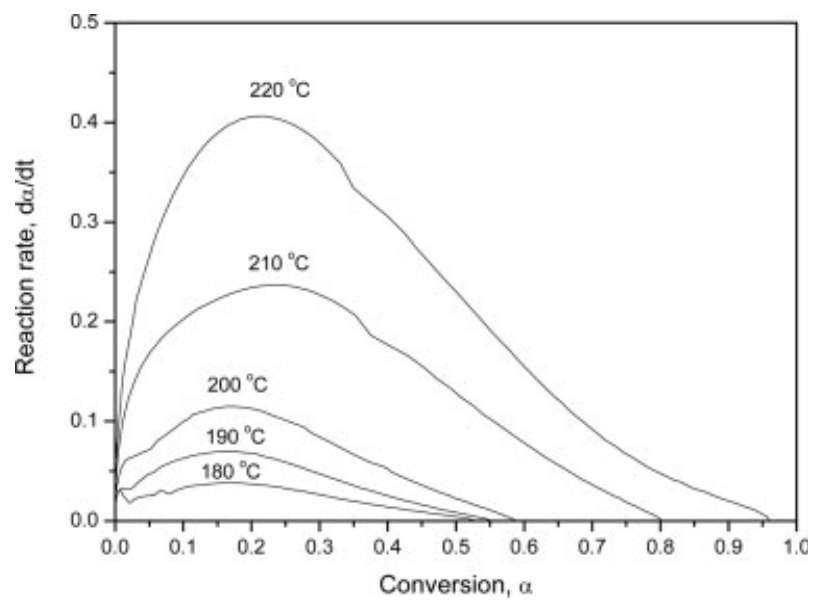

Figure 9. Plots of reaction rate as a function of conversion for the nanocomposites cured at different temperatures.

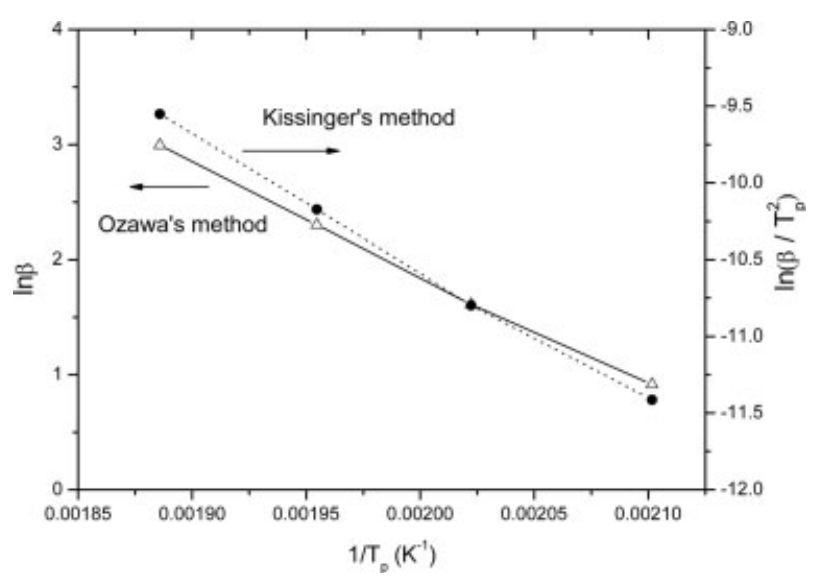

Figure 10. Representations of Ozawa's and Kissinger's methods of calculating the activation energy from nonisothermal data for pure PBZ $\left(T_{\mathrm{p}}\right.$, temperature at maximum reaction rate; $\beta$, heating rate).

require prior knowledge of the reaction mechanism to quantify the kinetic parameters.

\section{Kissinger Method}

This approach assumes that, for thermoset curing, the extent of the reaction at the peak exotherm is constant and independent of the heating rate. The value of $E_{\mathrm{a}}$ can be obtained as follows:

$$
-\frac{E_{\mathrm{a}}}{R}=\frac{\mathrm{d}\left[\ln \left(\beta / T_{\mathrm{p}}^{2}\right)\right]}{\mathrm{d}\left(1 / T_{\mathrm{p}}\right)}
$$

where $\beta$ is a heating rate, $T_{\mathrm{p}}$ is the maximum exothermic temperature, and $R$ is the universal gas constant. Plotting $\ln \left(\beta / T_{\mathrm{p}}^{2}\right)$ against $1 / T_{\mathrm{p}}$ provides the activation energy without requiring a specific assumption of the conversion-dependent function. We calculated the slope as indicated in Figure 10 . Table 1 lists all the values of $E_{\text {a }}$; the acti-

Table 1. Activation Energies Obtained Using the Kissinger and Ozawa Methods for PBZ/Clay Nanocomposites

\begin{tabular}{lccc}
\hline & & \multicolumn{2}{c}{$E_{\mathrm{a}}$} \\
\cline { 3 - 4 } Sample & $\begin{array}{c}\text { Clay } \\
\text { Content } \\
(\text { wt \% })\end{array}$ & $\begin{array}{c}\text { Kissinger } \\
(\mathrm{KJ} / \mathrm{mol})\end{array}$ & $\begin{array}{c}\text { Ozawa } \\
(\mathrm{KJ} / \mathrm{mol})\end{array}$ \\
\hline PBZ & 0 & 112 & 117 \\
PBZ/clay & 3 & 107 & 111 \\
PBZ/clay & 6 & 106 & 110 \\
PBZ/clay & 10 & 104 & 104 \\
PBZ/clay & 15 & 90 & 93 \\
\hline
\end{tabular}


Table 2. Results Obtained from Isothermal Experiments on the PBZ/Clay Nanocomposites

\begin{tabular}{|c|c|c|c|c|c|c|c|}
\hline Sample & $\begin{array}{l}\text { Clay Content } \\
(\text { wt } \%)\end{array}$ & $\begin{array}{c}\text { Curing } \\
\text { Temp. }\left({ }^{\circ} \mathrm{C}\right)\end{array}$ & $\begin{array}{l}\text { Rate Const. } \\
\quad k\left(\min ^{-1}\right)\end{array}$ & $\begin{array}{c}E_{\mathrm{a}} \\
(\mathrm{KJ} / \mathrm{mol})\end{array}$ & $m$ & $n$ & $m+n$ \\
\hline \multirow[t]{5}{*}{ PBZ } & 0 & 180 & 0.35 & 110 & 0.9 & 1.9 & 2.8 \\
\hline & & 190 & 0.54 & & & & \\
\hline & & 200 & 0.98 & & & & \\
\hline & & 210 & 2.14 & & & & \\
\hline & & 220 & 2.62 & & & & \\
\hline \multirow[t]{5}{*}{ PBZ/clay } & 3 & 180 & 0.29 & 104 & 0.6 & 2.0 & 2.6 \\
\hline & & 190 & 0.43 & & & & \\
\hline & & 200 & 0.62 & & & & \\
\hline & & 210 & 0.98 & & & & \\
\hline & & 220 & 1.87 & & & & \\
\hline \multirow[t]{5}{*}{ PBZ/clay } & 6 & 180 & 0.39 & 100 & 0.8 & 1.7 & 2.5 \\
\hline & & 190 & 0.42 & & & & \\
\hline & & 200 & 0.56 & & & & \\
\hline & & 210 & 1.13 & & & & \\
\hline & & 220 & 1.55 & & & & \\
\hline \multirow[t]{5}{*}{ PBZ/clay } & 10 & 180 & 0.37 & 105 & 0.5 & 2.1 & 2.6 \\
\hline & & 190 & 0.35 & & & & \\
\hline & & 200 & 0.61 & & & & \\
\hline & & 210 & 1.15 & & & & \\
\hline & & 220 & 2.82 & & & & \\
\hline \multirow[t]{5}{*}{ PBZ/clay } & 15 & 180 & 0.14 & 106 & 0.2 & 2.2 & 2.4 \\
\hline & & 190 & 0.41 & & & & \\
\hline & & 200 & 0.88 & & & & \\
\hline & & 210 & 1.71 & & & & \\
\hline & & 220 & 5.41 & & & & \\
\hline
\end{tabular}

vation energy is found to decrease upon increasing the clay content. In other words, higher clay content can decrease the activation energy and promote the ring opening polymerization.

\section{Ozawa Method}

The Ozawa method is another approach that is used widely for dynamic kinetic analysis without requiring any assumptions to be made regarding the conversion-dependence function. Its general expression may be represented by eq 4 :

$$
E_{\mathrm{a}}=\frac{-R}{1.052} \frac{\Delta \ln \beta}{\Delta\left(1 / T_{\mathrm{p}}\right)}
$$

Figure 10 provides plots of $\ln \beta$ as a function of (1/ $T_{\mathrm{p}}$ ), and from which we can obtain the values of the slope to calculate the activation energy. Table 1 also provides all of these values of $E_{\mathrm{a}}$; it indicates that the results obtained when using the Ozawa method are close to those provided by the Kissinger method.

\section{Isothermal Kinetic Analysis (Autocatalytic Model)}

The autocatalytic model is a phenomenological approach that was developed by Kamal. If a system is autocatalytic, the reaction rate can be described by the general expression:

$$
\frac{\mathrm{d} \alpha}{\mathrm{d} t}=k \alpha^{m}(1-\alpha)^{n}
$$

where $\alpha$ is the reaction conversion, $k$ is the kinetic rate constant, $m$ and $n$ are the kinetic exponents of the reaction, and $(m+n)$ is the overall reaction order.

In practice, however, the curing reaction will cease when the reaction conversion reaches its maximum value $\left(\alpha_{\max }\right)$. In this case, eq 5 must be corrected into the form of eq $6 .^{33-35}$

$$
\frac{\mathrm{d} \alpha}{\mathrm{d} t}=k \alpha^{m}\left(\alpha_{\max }-\alpha\right)^{n}
$$

when $\alpha=\alpha_{\max } \rightarrow \frac{\mathrm{d} \alpha}{\mathrm{d} t}=0 . \alpha_{\max }$ is based on different curing temperatures. Equations 7 and 8 are obtained after rearranging eq 6 and taking its 


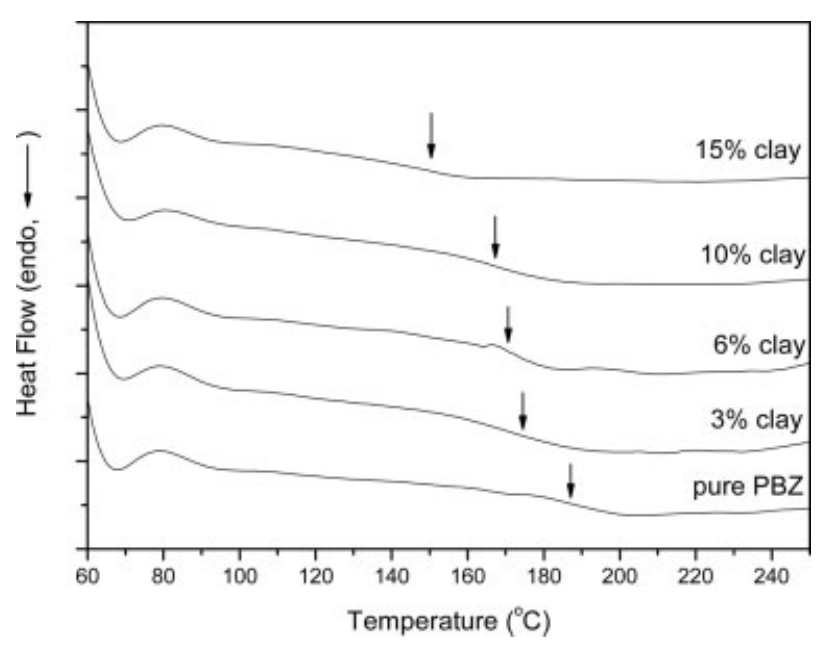

Figure 11. DSC curves for determining the glass transition temperatures of the nanocomposites.

natural logarithm. Using the iteration method allows the approximate values of $m, n$, and $k$ to be calculated according to eqs 7 and 8 . When $\left|n_{i+1}-n_{i}\right|<0.01$, the iteration is stopped and the values of $m, n$, and $k$ are obtained; Table 2 lists the results. The values of $(m+n)$ exist within the range between 2.4 and 2.8. The rate constants increase upon increasing the curing temperature.

$$
\begin{aligned}
\ln \left(\frac{(\mathrm{d} \alpha / \mathrm{d} t)}{\left(\alpha_{\max }-\alpha\right)^{n}}\right) & =\ln k+m \ln \alpha \\
\ln \left(\frac{(\mathrm{d} \alpha / \mathrm{d} t)}{k \alpha^{m}}\right) & =n \ln \left(\alpha_{\max }-\alpha\right)
\end{aligned}
$$

In addition, the rate constant ( $k$ ) may be described by the Arrhenius expression ${ }^{33}$ :

$$
k=A e^{-E_{\mathrm{a}} / R T}
$$

where $A$ is Arrhenius factor, $E_{\mathrm{a}}$ is the activation energy, $R$ is the universal gas constant, and $T$ is the absolute temperature. Taking the natural logarithm of eq 9 , we obtain eq 10 . Plotting $\ln k$ as a function of $1 / T$ for the different isothermal curing temperatures allows us to obtain the kinetic data, which are presented in Table 2. The activation energies of the PBZ/clay nanocomposites are smaller than that of pure PBZ.

$$
\ln k=\ln A-E_{\mathrm{a}} / R T
$$

Comparing the results of the three kinetic models, we find that the values of the activation energies are quite close. Hence, it is possible to calculate the activation energy and kinetic parameters in this system-including the values of $m, n$, and $k$ - by using any one of the Kissinger, Osawa, or Kamal methods.

\section{Analyzing Glass Transition Temperatures}

Figure 11 displays the DSC thermograms of pure PBZ and the PBZ/clay nanocomposites; Table 3 lists the data. The glass transition temperature, $T_{\mathrm{g}}$, decreases upon increasing the clay content. The main reason for this phenomenon is that the conversion of PBZ decreases upon increasing the clay content. Figure 8 indicated that reaching a high value for the conversion was difficult at high clay content. In other words, the value of $T_{\mathrm{g}}$ is lower when the conversion is low.

\section{Thermal Stability of PBZ/Clay Nanocomposites}

We performed TGA of the MBM-clay based nanocomposites to monitor the effect that dispersion of the clay nanolayer in the PBZ matrix has on the thermal properties. Figure 12 displays the TGA traces of pure PBZ and the PBZ/clay nanocomposites. The PBZ/clay nanocomposites displayed higher decomposition temperatures than

Table 3. TGA and DSC Data for PBZ/Clay Nanocomposites

\begin{tabular}{lccccc}
\hline & & & \multicolumn{2}{c}{ Weight Loss Temperature $\left({ }^{\circ} \mathrm{C}\right)$} & \\
Sample & $\begin{array}{c}\text { Clay } \\
(\text { wt } \%)\end{array}$ & $\begin{array}{c}T_{\mathrm{g}} \\
\left({ }^{\circ} \mathrm{C}\right)\end{array}$ & 5 wt $\%$ & 50 wt $\%$ & $\begin{array}{c}\text { Char } \\
\text { Yield }(\%)\end{array}$ \\
\hline PBZ & 0 & 185 & 315 & 450 & 32 \\
PBZ/clay & 3 & 172 & 317 & 464 & 31 \\
PBZ/clay & 6 & 169 & 323 & 497 & 37 \\
PBZ/clay & 10 & 166 & 331 & 523 & 49 \\
PBZ/clay & 15 & 151 & 297 & 734 & 49 \\
\hline
\end{tabular}


did pure PBZ; that is the thermal stability of the nanocomposites was improved by the presence of dispersed clay nanolayers, which act as barriers to minimize the permeability of the volatile degradation products from the material. ${ }^{36}$ Table 3 summarizes the TGA results for these nanocomposites. From the temperatures of the 5 and 50\% weight losses, the TGA results of the PBZ/clay samples indicate a clear trend of improved thermal stability upon increasing the clay content. The PBZ/clay nanocomposites obviously have greater char yield, which increases upon increasing the clay content, as expected. The increase in char yield suggests the reduction of the polymer's flammability. ${ }^{37}$ The char is composed of aromatic compound after high temperature.

\section{CONCLUSIONS}

In this study, we successfully synthesized the intercalation agent MBM and prepared PBZ/clay hybrid nanocomposites. The sample had a mixture of exfoliated and intercalated structures at $3 \%$ clay content. The MBM intercalating agent, which contains a benzoxazine functional group that can undergo ring opening polymerization within the galleries of the clay, promotes the exfoliated structure of nanocomposite. DSC traces indicated that the onset of the ring opening of the benzoxazine unit in the presence of clay to form PBZ/clay nanocomposites occurred at relatively lower temperatures relative to that of the pristine benzoxazine; this finding suggests that the clay surface has a catalytic effect on the ring opening polymerization. Both the Kissinger and Ozawa methods of calculating the activation energy gave fairly consistent results; the activation energy decreased upon increasing the clay content as a result of a lower conversion. The Kamal method, based on an autocatalytic model, suggested that the total order of the reaction was between 2.4 and 2.8. The glass transition temperature $\left(T_{\mathrm{g}}\right)$ is decreased upon increasing the clay content. TGA traces indicated that the nanocomposites have higher thermal stabilities relative to that of the pristine PBZ. The char yields increased upon increasing the clay content.

\section{REFERENCES AND NOTES}

1. Riess, G.; Schwob, J. M.; Guth, G.; Roche, M.; Laude, B. In Advances in Polymer Science Syn- thesis; Culbertson, B. M.; McGrath, J. E., Eds.; Plenum: New York, 1985.

2. Ning, X.; Ishida, H. J Polym Sci Part A: Polym Chem 1994, 32, 1121.

3. Dunkers, J.; Ishida, H. Spectrochim Acta 1995, 51,855 .

4. Ishida, H.; Allen, D. Polymer 1996, 37, 4487.

5. Shen, B. S.; Ishida, H. J Appl Polym Sci 1996, 61, 1595.

6. Ishida, H.; Allen, D. J. J Polym Sci Part B: Polym Phys 1996, 34, 1019.

7. Takeichi, T.; Komiya, I.; Takayama, Y. Kyoka Purasutikkusu 1997, 43, 109 (in Japanese).

8. Usuki, A.; Kawasumi, M.; Kijima, Y.; Okada, A.; Kamigaito, O. J Mater Res 1993, 8, 1174.

9. Kojima, Y.; Usuki, A.; Kawasumi, M.; Okada, A.; Kurauchi, T.; Kamigaito, O. J Polym Sci Part A: Polym Chem 1993, 31, 1755.

10. Yano, K.; Usuki, A.; Okada, A.; Kurauchi, T. J Polym Sci Part A: Polym Chem 1993, 31, 2493.

11. Usuki, A.; Koiwai, A.; Kojima, Y.; Kawasumi, M.; Okada, A.; Kurauchi, T.; Kamigaito, O. J Appl Polym Sci 1995, 55, 1193.

12. Kawasumi, M.; Hasegawa, N.; Kato, M.; Kojima, Y.; Usuki, A.; Okada, A. Macromolecules 1997, 30, 6333.

13. Moet, A.; Akelah, A. Mater Lett 1993, 18, 97.

14. Moet, A.; Akelah, A.; Salahuddin, N.; Hiltner, A.; Baer, E. Mater Res Soc Symp Proc 1994, 351, 163.

15. Akelah, A. In Polymers and Other Advanced Materials: Emerging Technologies and Business Opportunities; Prasad, P. N.; Mark, J. E.; Tung, J. F., Eds.; Plenum: New York, 1995.

16. Giannelis, E. P. Adv Mater (Weinheim, Ger) 1996, 8, 29.

17. Giannelis, E. P. Annu Tech Conf Soc Plast Eng 1996, 54, 2998.

18. Giannelis, E. P. In Biomimetric Materials Chemistry; Mann, S., Ed.; VCH: New York, 1996.

19. Giannelis, E. P. Appl Organomet Chem 1998, 12 , 675.

20. Lan, T.; Pinnavaia, T. J. Chem Mater 1994, 6, 2216.

21. Wang, M. S.; Pinnavaia, T. J. Chem Mater 1994, $6,468$.

22. LeBaron, P. C.; Wang, Z.; Pinnavaia, T. J. Appl Clay Sci 1999, 15, 11.

23. Yang, Y.; Zhu, Z.; Yin, J.; Wang, X.; Qi, Z. Polymer 1999, 40, 4407.

24. Agag, T.; Koga, T.; Takeichi, T. Polymer 2001, 42, 3399.

25. Agag, T.; Takeichi, T. Polymer 2000, 41, 7083.

26. Horie, K.; Mita, I.; Kambe, H. J Polym Sci Polym Chem Ed 1970, 8, 2839.

27. Prime, R. B. In Thermal Characterization of Polymeric Materials; Turi, E. A., Ed.; Academic Press: New York, 1981; pp 425. 
28. Nam, J. D.; Seferis, J. C. J Appl Polym Sci 1993, $50,1555$.

29. Barral, L.; Cano, J.; Lopez, I.; Bueno, L.; Nogueira, P.; Abad, M. J.; Ramirez, C. J Polym Sci Part B: Polym Phys 2000, 38, 351.

30. Kissinger, H. E. Anal Chem 1957, 29, 1702.

31. Ozawa, T. J Therm Anal 1970, 2, 301.

32. Kamal, M. R. Polym Eng Sci 1974, 27, 782.

33. Fischer, M.; Tran, C. D. Anal Chem 1999, 71, 953.
34. Cole, R. C. Macromolecules 1991, 24, 3093.

35. Cole, K. C.; Hechler, J. J.; Nobel, D. Macromolecules 1991, 24, 3098.

36. Frischer, H. R.; Gielgens, L. H.; Koster, T. P. M. Acta Polym 1999, 50, 122.

37. Gilman, J. W.; VanderHart, D. L.; Kashiwagi, T. In Fire and Polymer; Nelson, G. L., Ed. American Chemical Society: Washington, DC, 1995; ACS Symposium Series, p 161. 